\title{
Review Article \\ CD147 as a Novel Prognostic Biomarker for Hepatocellular Carcinoma: A Meta-Analysis
}

\author{
Fei Peng, ${ }^{1}$ Hui Li, ${ }^{2}$ Qian You, ${ }^{1}$ Hongru Li, ${ }^{3}$ Dongwen Wu, ${ }^{3}$ Chunxiang Jiang, \\ Guangtong Deng, ${ }^{4}$ Yan $\mathrm{Li}^{5}{ }^{5}$ Yuyan $\mathrm{Li},{ }^{5}$ and $\mathrm{Yi} \mathrm{Wu}^{1}$
${ }^{1}$ Department of Laboratory, The First Affiliated Hospital of Hunan Normal University, Hunan Provincial People's Hospital, Changsha, Hunan, China
${ }^{2}$ Reproductive Department, Xiangya Hospital, Central South University, Changsha, China
${ }^{3}$ Xiangya School of Medicine, Central South University, Changsha, China
${ }^{4}$ Hepatic Surgery Department of Xiangya Hospital, Central South University, Changsha, China
${ }^{5}$ Department of Pediatrics, The First Affiliated Hospital of Hunan Normal University, Hunan Provincial People's Hospital, Changsha, Hunan, China

Correspondence should be addressed to Yi Wu; wuyi19701210@outlook.com

Received 18 November 2016; Accepted 19 December 2016; Published 12 March 2017

Academic Editor: Hannes Stockinger

Copyright (C) 2017 Fei Peng et al. This is an open access article distributed under the Creative Commons Attribution License, which permits unrestricted use, distribution, and reproduction in any medium, provided the original work is properly cited.

\begin{abstract}
We conducted a meta-analysis to investigate the controversial association of CD147 expression with HCC prognosis and clinicopathological characteristics. Eight studies from PubMed (1966-2016), EMBASE (1980-2016), Cochrane Library (19962016), Web of Science (1945-2016), China National Knowledge Infrastructure (1982-2016), and Wanfang databases (1988-2016) were considered. The associations between CD147 expression and clinicopathological parameters and overall survival (OS) or DFS/RFS were reassessed using the meta-analysis for odds ratio (OR) or hazard ratio (HR) and 95\% confidence interval (CI). CD147 expression was associated with DFS/RFS (HR $=3.26$; 95\% CI: $1.82-5.83 ; P<0.0001)$ but not with OS (HR = 1.35; 95\% CI: $0.56-3.29 ; P=0.51)$. We also delved deeper into the association between median survival time and CD147 expression owing to significant heterogeneity and found significant differences between high and low CD147 expression groups with respect to median survival time. CD147 expression was closely associated with the TNM stage (OR $=0.18 ; 95 \% \mathrm{CI}: 0.04-0.85 ; P=0.03)$ and venous invasion $(\mathrm{OR}=6.29 ; 95 \% \mathrm{CI}: 1.70-23.20 ; P=0.006)$. In contrast, there was no association between CD147 expression and tumor stage, cirrhosis, differentiation, lymph node metastasis, HBsAg, and serum AFP levels. Thus, CD147 expression is potentially closely related to HCC survival and associated clinicopathological parameters, paving the way for further research.
\end{abstract}

\section{Introduction}

Hepatocellular carcinoma (HCC) is the sixth most prevalent malignancy and the third leading cause of cancer-related death worldwide [1]. Although incidence rates have been declining for most cancers, rates are increasing for HCC [2]. In spite of its increased incidence, there is only basic understanding of disease pathogenesis and there are limited therapeutic options [3]. The 5-year overall survival rate of individuals with $\mathrm{HCC}$ is only $8.9 \%$, and this has barely improved over the past two decades [4]. Recently, many prognostic markers, such as CD133, CD44, keratin 19, Serum $\mathrm{M} 65$, and serum sCD163, have been introduced to help identify patients who are likely to have a poor prognosis and benefit from more aggressive treatment approaches [5-8].

CD147 is also known as HAb18G in humans $[9,10]$. As a transmembrane glycoprotein and a member of immunoglobulin superfamily, it was first named as tumor cell-mediated collagen enzyme activation factor (tumor cell collagenase stimulatory factor, TCSF) and later on renamed as EMMPRIN [11]. Earlier studies demonstrated that the CD147 molecule was highly expressed on the surface of various cancer cells, including cancers of the liver, lung, breast, kidney, colon, prostate, and esophagus [12]. There is emerging evidence indicating that CD147 plays a central role in the progression of many cancers due to increased adhesion, 
migration, invasion, and matrix metalloproteinases [1316]. Importantly, increased expression of cancer-associated CD147 predicts aggressive behavior and poor prognosis [12, 17-20].

Recent reports have indicated that the expressions of CD147 correlate with poor clinical factors and outcomes in hepatic carcinoma [21]. However, another study has nullified this hypothesis [18]. Therefore, we conducted this metaanalysis for the quantitative inspection of the relationship between CD147 expression and clinicopathological features and survival of hepatic carcinoma patients.

\section{Materials and Methods}

The following were the criteria for the inclusion of studies in our analysis.

(1) The studies had to be published or unpublished case control study or cohort study in English or Chinese with the full text available. (2) All cases had complete clinicopathological characteristic data, without radiotherapy or chemotherapy or biological therapy before sampling. (3) Diagnosis of hepatic carcinoma cancer was proven by pathological methods. (4) Studies must have CD147 expression analyzed by immunohistochemical staining in primary hepatic carcinoma tissue (via either biopsy or surgical) and not in serum or any other kind of specimen. (5) The best quality study was retained for conducting duplicated study.

The following were the criteria for the exclusion of studies in our analysis: (1) cell or animal studies, case reports, letters, and reviews; (2) the standard of pathological diagnosis being not clear.

2.1. Search Strategy. The studies were included from PubMed (1966-2016), EMBASE (1980-2016), Cochrane Library (1996-2016), Web of Science (1945-2016), China National Knowledge Infrastructure (1982-2016), and Wanfang databases (1988-2016). The studies were restricted to humans, but not by date, language, or publication status. The following combined search terms were used: (Liver Neoplasms OR hepatic neoplasm* OR hepatocellular cancer* OR hepatic cancer* OR liver cancer*) AND (CD147 OR extracellular matrix metalloproteinase inducer OR EMMPRIN) AND (prognosis OR survival OR outcome OR prognostic). We combined the terms appropriately with $\mathrm{MeSH}$ Terms and used an appropriate adjustment for different databases. Details of the search strategies can be found in Appendix 1 (see Supplementary Material available online at https://doi.org/10.1155/2017/5019367).

2.2. Statistical Analysis. The records were independently scanned by two authors to exclude irrelevant studies. Then, full-text articles were independently excluded, and controversial opinions were resolved by the third author. All of the data were extracted independently by two authors. The NewcastleOttawa Scale (NOS) [22] was applied to assess the included studies. RevMan 5.3 software and Stata 13.0 software were used for analysis. For each study, the HR was estimated by a method that was dependent on the results provided in the publication. The most accurate method was to retrieve the
HR estimate and its variance from the reported results or to calculate it directly using parameters provided by the authors for univariate analysis. If an article described both univariate and multivariate factors, we chose the latter as the survival in HCC is affected by a combination of factors. Otherwise, Kaplan-Meier curves were read using Engauge Digitizer version 4.1 [23], which can estimate a relatively accurate $H R$ $[24,25]$, with the assumption that, during the study follow-up, the rate of patients censored was constant. If this method was used, three independent persons read the curves to reduce the variation. Hazard ratios (HR) and 95\% confidence intervals (95\% CI) were used to evaluate the relationship between CD147 expression among OS (overall survival) and DFS/RFS (disease-free survival/recurrence-free survival). Median survival ratio (MSR) and 95\% CI were used to evaluate the median survival time. Moreover, we also examined the correlation between CD147 expression and the clinical variables in liver cancer through odds ratio (OR). Fixed-effects model was adopted for studies without significant heterogeneity $\left(P>0.1\right.$ and $\left.I^{2}<50 \%\right)$; otherwise, random-effects model was applied. Wherever possible, heterogeneity was explored and subgroup analyses were performed according to followup time, the nature of HR (multivariate or univariate), liver transplantation status, and cut-off value. These aspects may influence our conclusion about the association between CD147 and survival of patients with HCC.

Sensitivity analysis was performed to evaluate the influences of individual studies on the final effect size. Egger's test was used to assess publication bias $(P<0.05$ was considered statistically significant). If publication bias was confirmed and the data were enough, a trim-and-fill method developed by Duval and Tweedie was implemented to adjust for this bias [26]. Then, we replicated the funnel plot with its "missing" counterparts around the adjusted summary estimate.

\section{Results}

3.1. Study Characteristics. A total of 202 studies were identified, and 120 studies were excluded because of duplication. Figure 1 illustrates the trial flow chart. After reading the titles and abstracts, 48 studies were excluded. Thirty-four full-text studies were carefully reviewed (excluded for being animal studies [ $n=3]$, serum CD147 expression [ $n=2]$, no survival data $[n=8]$, and being completely irrelevant $[n=11])$. A total of 10 studies $[18,21,26-31]$ were identified for qualitative analysis. The study by Ji et al. [32] did not provide the data of HR and 95\% CI for HCC patients, which only know mean survival time of recurrence-free survival (RFS); another study (W-C Tsai) [33] does not provide the cut-off value for judging CD147 positive expression. After selection, 8 studies with 880 patients were finally used for analysis of the prognostic value of CD147 expression in the meta-analysis. All 8 studies adopted immunohistochemistry (IHC) as the detection method, but the method for judging negative and positive staining was different among them. In addition, all patients in eight studies were diagnosed with HCC (hepatocellular carcinoma) and were of Asian origin. Four studies reported OS (overall survival), four studies provided DFS/RFS (diseasefree survival/recurrence-free survival), three studies reported 

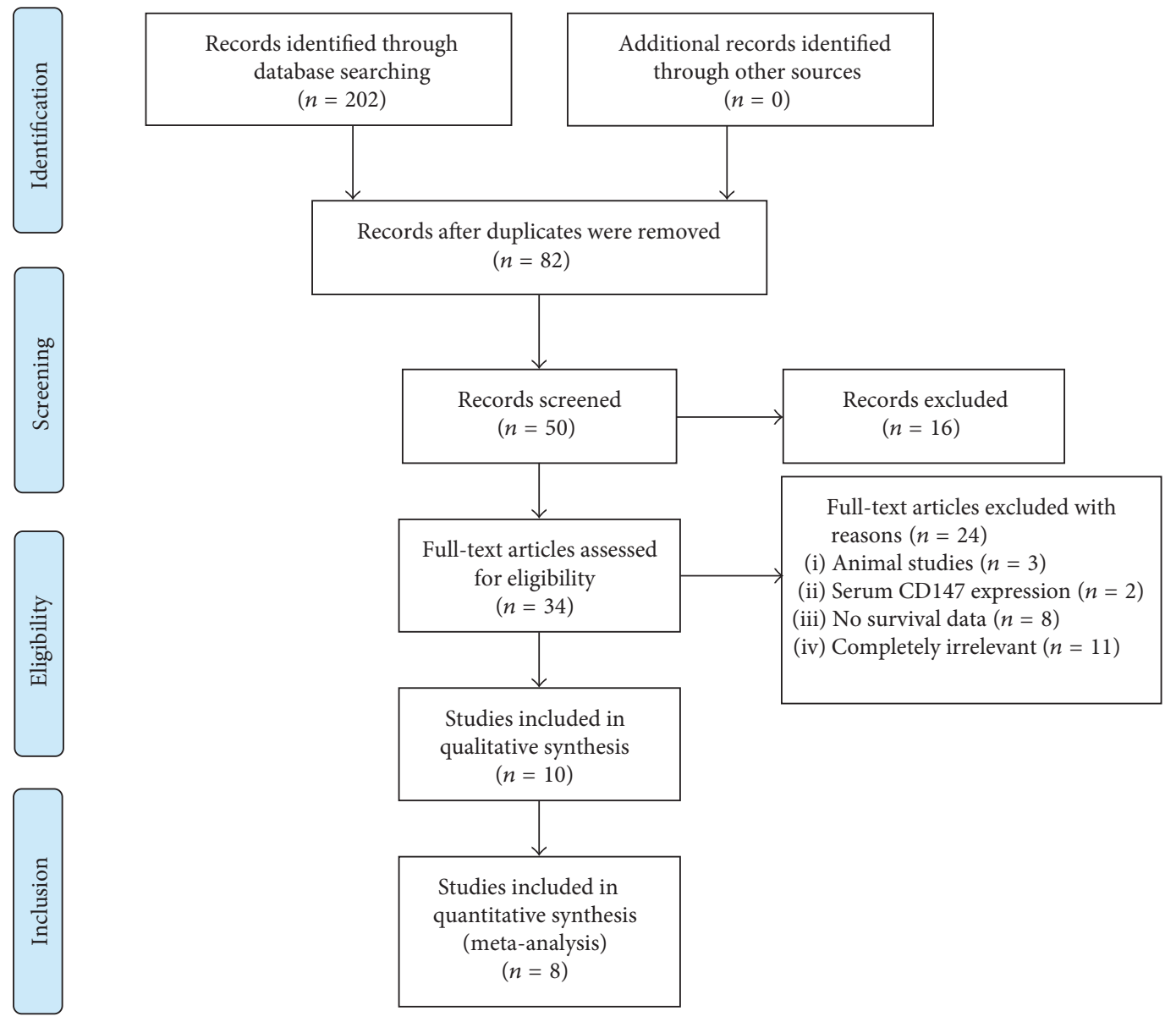

FIGURE 1: Flow chart of the selection process.

median survival time, seven studies provided follow-up time, three articles contained HR from multivariate factors, and five articles provided survival curves. One of the two articles that talked about liver transplantation had the sample taken before the transplantation without radiotherapy and chemotherapy, whereas the other had studied specimens from liver cancer patients with cirrhosis. All samples from these two studies were confirmed for HCC by histological studies. Table 1 lists the major characteristics of the selected studies; we used the NOS scale to evaluate the literature, and all of the studies had a score greater than 5, indicating that the quality of the literature was high. Moreover, we performed a subgroup analysis according to four aspects: follow-up time more than 5 years, HR from multivariate or univariate analysis, with or without liver transplantation, and cut-off value (more than $10 \%$ of cells stained). The characteristics of the studies are presented in Table 1 and the NOS results are presented in Table 2.

3.2. Correlation between CD147 Expression and OS. Four [18, $29,30,34]$ OS-related pieces of data displayed heterogeneity $\left(I^{2}=83 \% ; P=0.0006\right)$ and random model showed that high CD147 expression was not significantly associated with poor OS, as compared to low CD147 expression
$(\mathrm{HR}=1.35 ; 95 \% \mathrm{CI}: 0.56-3.29 ; P=0.51)$. In addition, we conducted subgroup analysis according to follow-up time, HR from multivariate or univariate analysis, with or without liver transplantation, and cut-off value (Table 3 ). In the univariate/multivariate subgroup analysis, heterogeneity was considerably dissolved in the univariate analysis group $\left(I^{2}\right.$ $=48 \% ; P=0.15)$. Moreover, there was a close association between OS and CD147 expression ( $\mathrm{HR}=2.21$; 95\% CI: 1.44-3.38; $P=0.0003$ ) (Figure 2), but this result could not have enough persuasion due to the limitation of subgroup analysis. However, there were no significant differences in the subgroups of follow-up time more than 5 years $(\mathrm{HR}=1.75$; 95\% CI: $0.57-5.34 ; P=0.33$ ) and without liver transplantation $(\mathrm{HR}=1.35$; 95\% CI: $0.56-3.29 ; P=0.51)$. In addition, sensitivity analysis indicated that the result was stable (Figure 3).

3.3. Correlation between CD147 Expression and DFS/RFS. Four studies [21, 27, 28, 31] demonstrated the association of CD147 expression with DFS/RFS. The combined data showed significant association between high CD147 expression and DFS/RFS (HR = 3.26; 95\% CI: 1.82-5.83; $P<0.0001)$ without heterogeneity $\left(I^{2}=34 \% ; P=0.21\right.$ ) (Figure 4 ). Sensitivity analysis showed that our results were unstable (Figure 5). In 


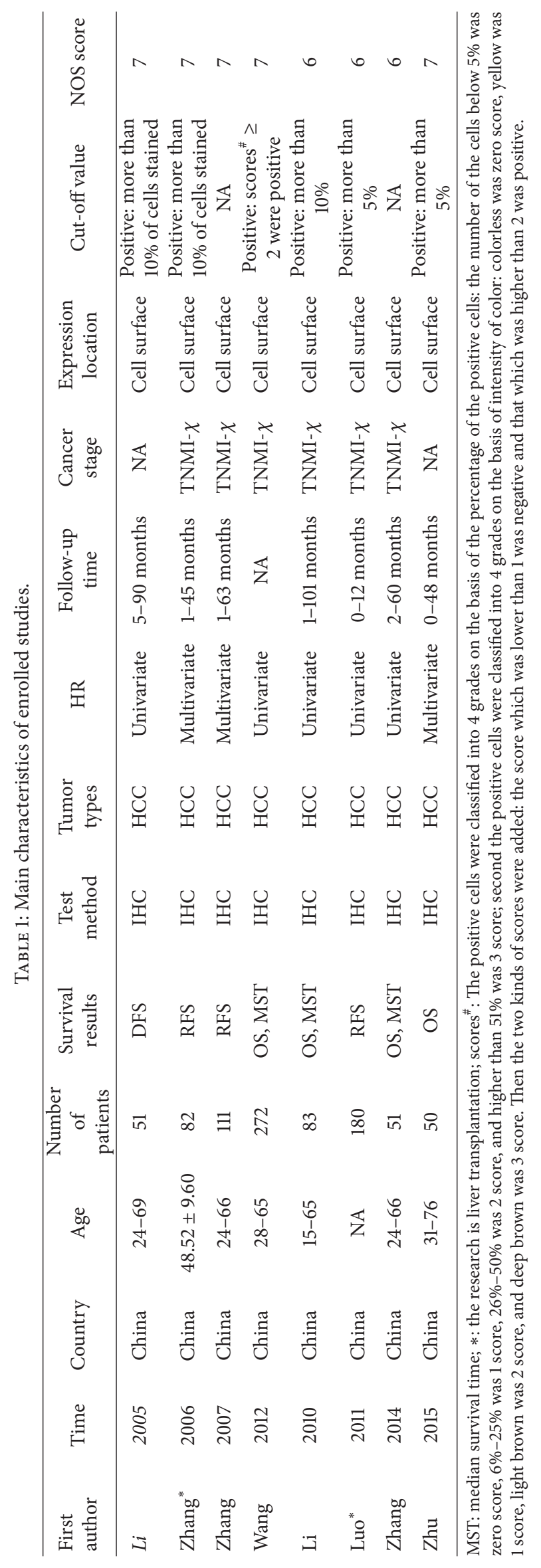




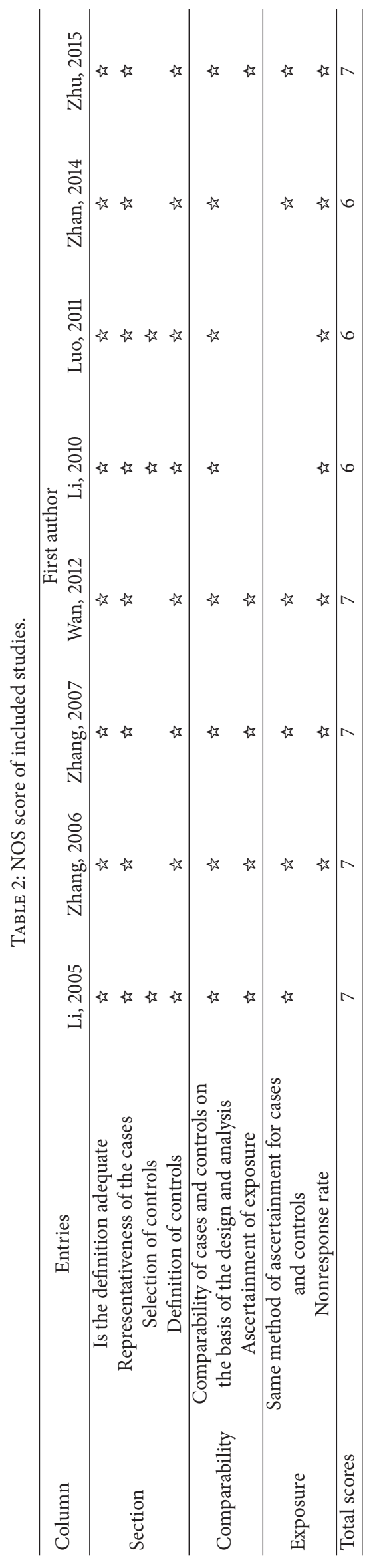




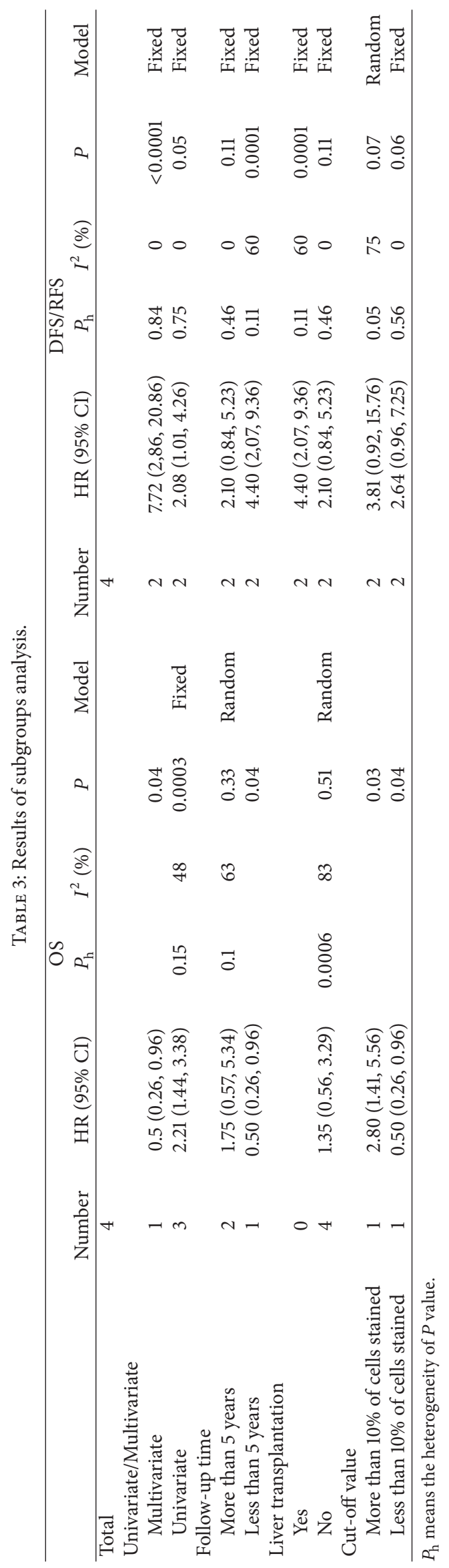




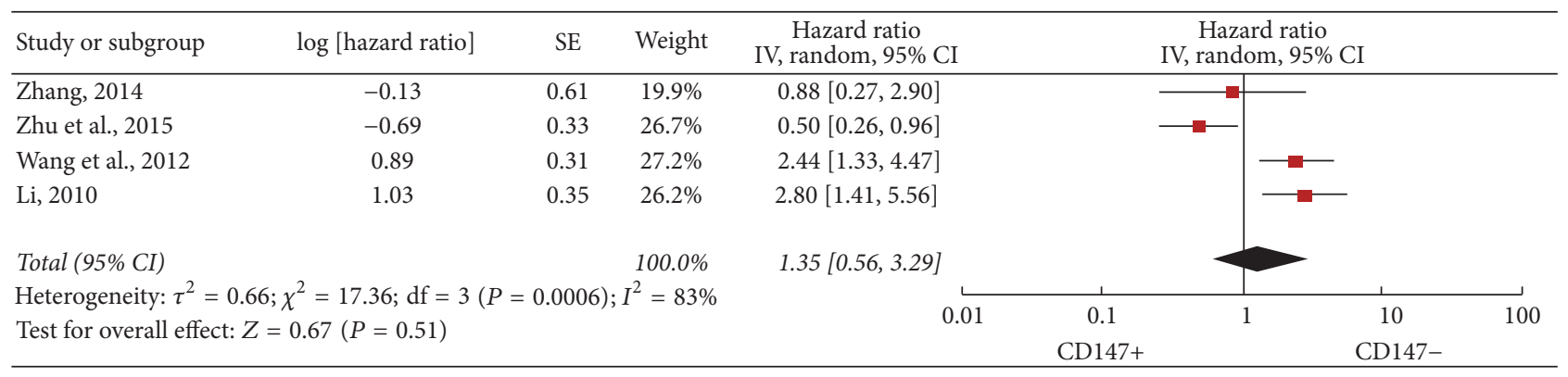

FIgURE 2: Forest plot of HR of OS for patients with HCC.

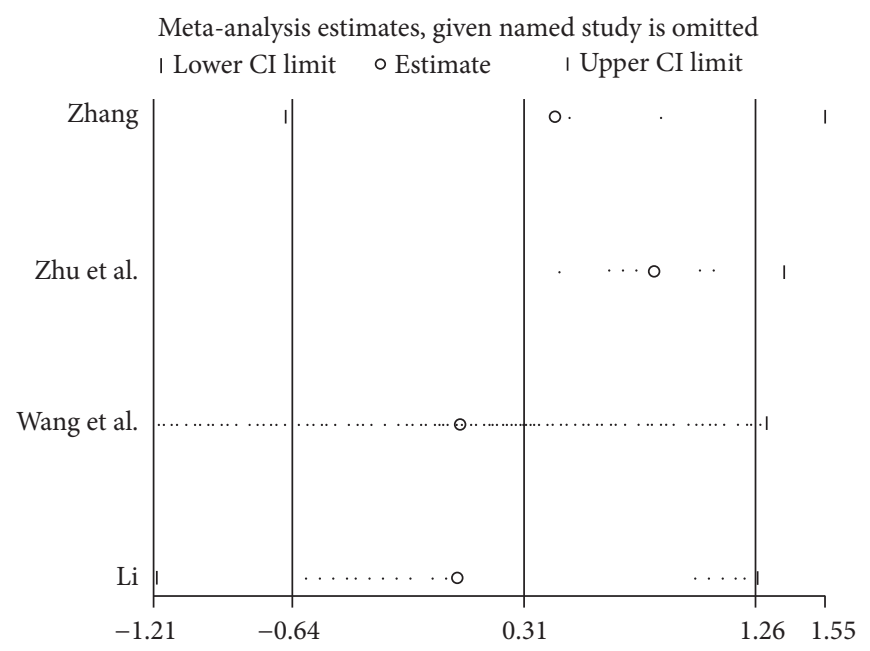

FIgUre 3: Sensitive analysis of OS for patients with HCC.

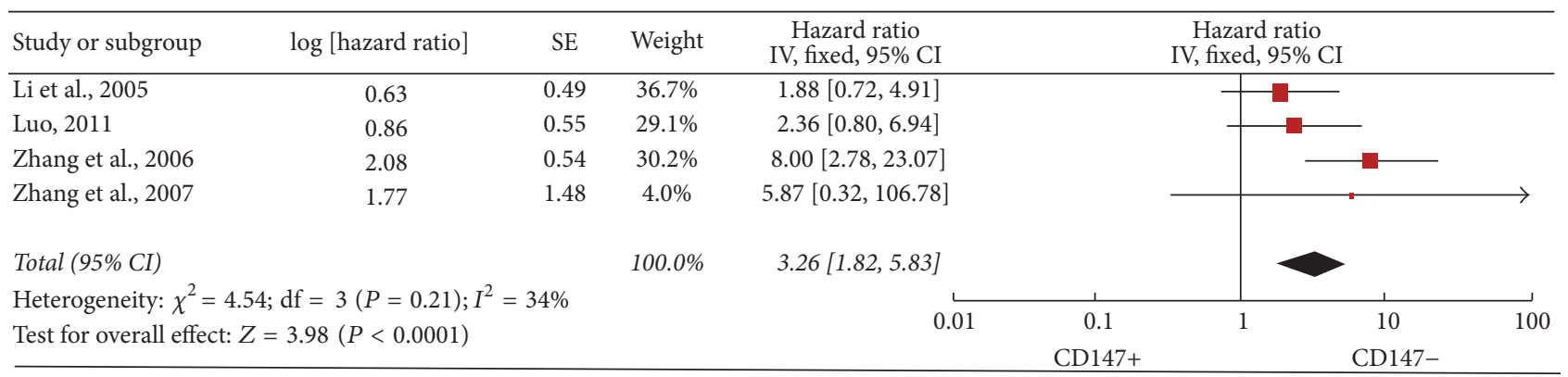

FIGURE 4: Forest plot of HR for DFS/RFS of patients with HCC.

addition, subgroup analysis indicated that there is significant difference in the groups of multivariate analysis, follow-up time less than 5 years, and with liver transplantation (Table 3 ). Thus, the association of CD147 expression with DFS/RFS of patients with HCC is speculative.

3.4. Correlation between CD147 Expression and Median Survival Time. Three studies [29, 30, 34] were chosen for analyzing the relationship between CD147 expression and median survival time in patients with HCC. There was significant association of high CD147 expression with median survival time $(\mathrm{MSR}=0.336 ; 95 \% \mathrm{CI}: 0.224-0.504 ; P=$
$0.000)$ with significant heterogeneity $\left(I^{2}=92.1 \% ; P=0.000\right)$ (Appendix 2). Owing to the significant heterogeneity and the fact that only three studies were included, we also made a description of the results. Median survival times reported by Wang et al. [29], Li [30], and Zhang [34] were 24 months, 14 months, and 10 months, respectively, in high CD147 expression group. All these Zhang et al.s studies have significant difference between the high CD147 expression and low CD147 expression groups with respect to median survival time. Therefore, the conclusion that high CD147 expression group has a shorter median survival time than low CD147 expression is speculative. 


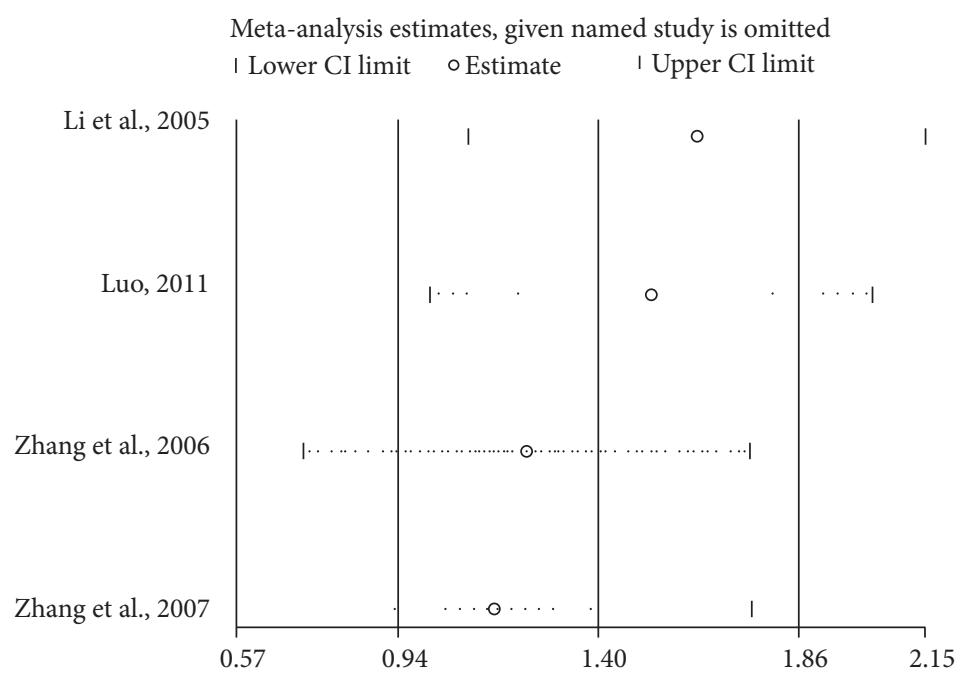

FIGURE 5: Sensitive analysis for DFS/RFS of patients with HCC.

TABLE 4: Meta-analyses of CD147 Expression classified by clinicopathological parameters.

\begin{tabular}{lccccc}
\hline Variables & Number of studies & Model & OR $(95 \% \mathrm{CI})$ & $P$ value & Heterogeneity $\left(I^{2}, P\right.$ value $)$ \\
\hline Tumor size $(\leq 5 \mathrm{~cm} />5 \mathrm{~cm})$ & 5 & Random & $0.38(0.14,1.03)$ & 0.06 & $88 \%,<0.00001$ \\
Cirrhosis (absent/present) & 4 & Random & $0.44(0.13,1.45)$ & 0.18 & $60 \%, 0.06$ \\
Differentiation (well/middle or low) & 5 & Random & $0.54(0.15,1.96)$ & 0.35 & $83 \%,<0.0001$ \\
TMNI- $\alpha / \beta-\chi$ & 6 & Random & $0.18(0.04,0.85)$ & 0.03 & $91 \%,<0.00001$ \\
Lymph node metastasis (yes/no) & 2 & Fixed & $3.60(0.96,13.53)$ & 0.06 & $0 \%, 0.60$ \\
HBsAg (positive/negative) & 4 & Fixed & $1.06(0.64,1.74)$ & 0.82 & $31 \%, 0.23$ \\
Venous invasion (present/absent) & 2 & Random & $6.29(1.70,23.20)$ & 0.006 & $74 \%, 0.05$ \\
Serum AFP level $(\leq 25 / \geq 25 \mu \mathrm{g} / \mathrm{L})$ & 3 & Random & $0.28(0.04,1.89)$ & 0.19 & $85 \%, 0.001$ \\
\hline
\end{tabular}

3.5. Correlation between CD147 Expression and Clinicopathological Parameters. Based on the ORs derived from each available study, we also evaluated the correlation between CD147 expression and some clinical characteristics, including tumor size, cirrhosis, differentiation, the TNM stage, lymph node metastasis, HBsAg, venous invasion, and serum AFP level. The results showed that CD147 expression was associated with the TNM stage $(\mathrm{OR}=0.18 ; 95 \% \mathrm{CI}$ : 0.04-0.85; $P=$ $0.03)$ and venous invasion $(\mathrm{OR}=6.29 ; 95 \% \mathrm{CI}: 1.70-23.20$; $P=0,006$ ) (Table 4). However, there were no significant differences between CD147 expression and any other clinical characteristics (Figure 6).

\section{Publication Bias}

The publication bias of the included studies was evaluated through Egger's tests. The corresponding $P$ values of OS and DFS/RFS were 0.782 (Appendix 3) and 0.608 (Appendix 4), respectively, indicating that the meta-analysis did not display publication bias.

\section{Discussion}

Hepatocellular carcinoma (HCC) is the most common primary liver cancer and the second most frequent cause of cancer-related death worldwide [35]. Caudron et al. reported that CD147 expression and ulceration status contributed to the overall survival of patients with cutaneous melanoma [36]. In addition, Bauman et al. demonstrated that membrane-associated CD147 expression was associated with tumor progression [37].

Our meta-analysis is the first one to investigate the association between CD147 expression and the survival rate of patients with liver cancer. A total of 880 patients with HCC were included in our meta-analysis. Our results indicated that there was no significant difference between CD147 expression and OS. However, the results differed among the subgroups of univariate analysis, which showed a close association between CD147 expression and OS. Tsai et al. [33] also demonstrated that CD147 expression was closely related to OS in univariate analysis. Moreover, low CD147 expression was related to longer survival. There exist conflicting views like those of $\mathrm{Zhu}$ et al. [18] who provided evidence that patients with HCC with high CD147 expression have longer survival. Our analyses also proved that CD147 expression in HCC is associated with DFS/RFS. This is contradictory to the report by $\mathrm{Li}$ [30], which showed that there was no significant difference in disease-free survival between high and low CD147 expression groups. According to our subgroup analysis, we found close association between CD147 expression and DFS/RFS in the 


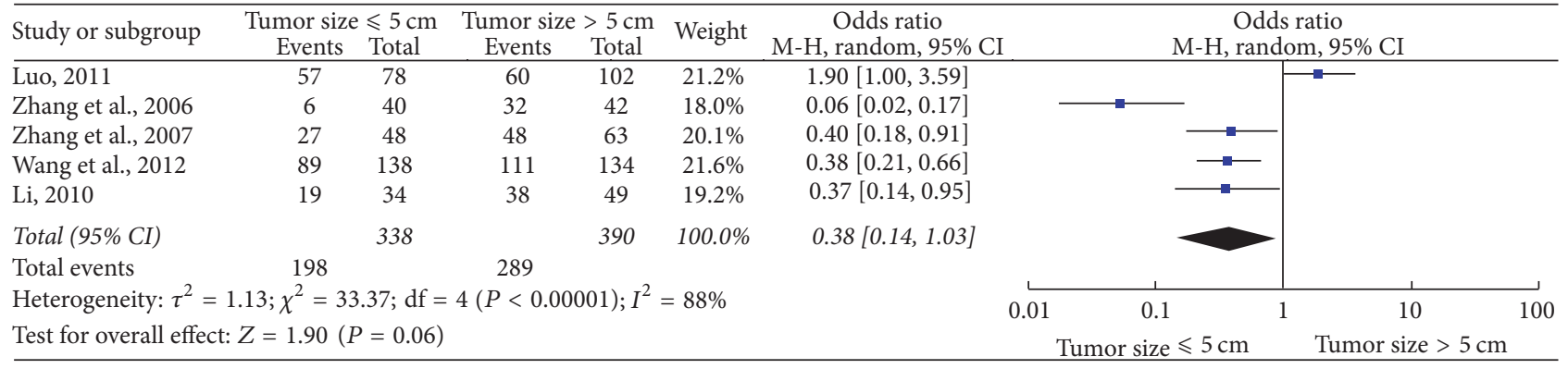

(a)

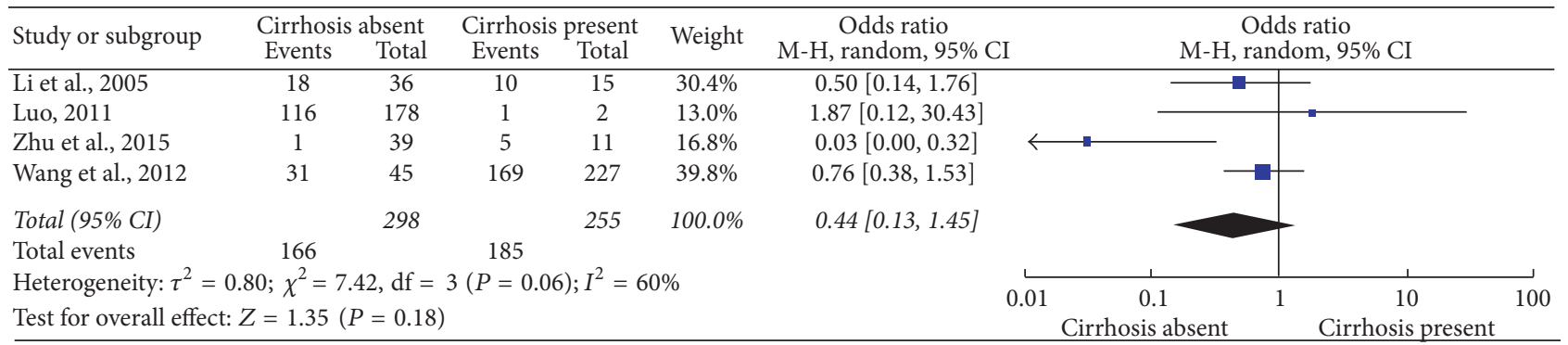

(b)

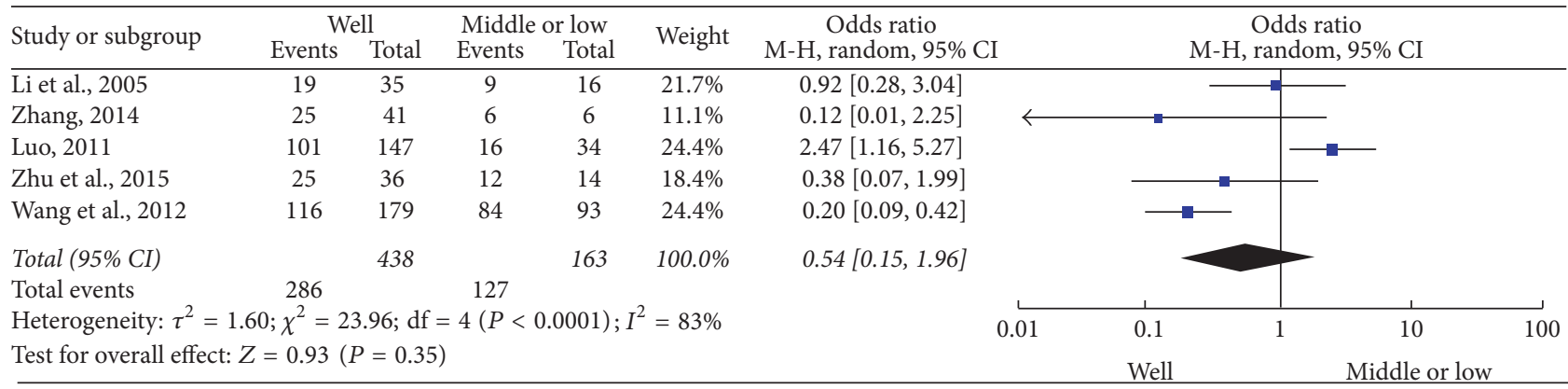

(c)

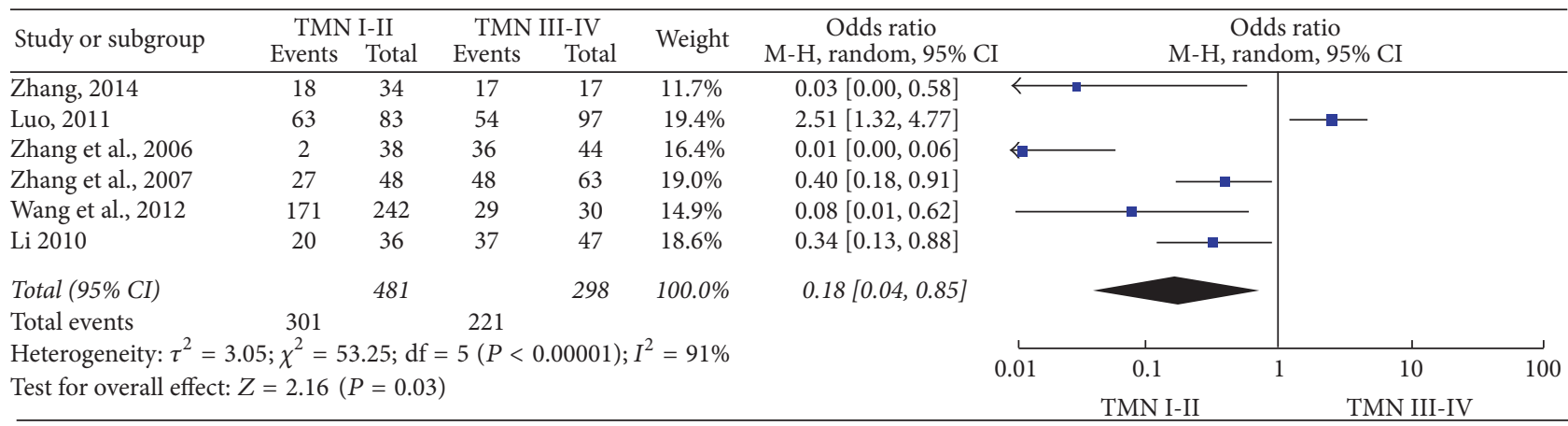

(d)

\begin{tabular}{|c|c|c|c|c|c|c|c|c|c|c|c|}
\hline \multirow{2}{*}{ Study or subgroup } & \multicolumn{2}{|c|}{$\begin{array}{l}\text { Lymph node } \\
\text { metastasis }\end{array}$} & \multicolumn{2}{|c|}{$\begin{array}{l}\text { No lymph node } \\
\text { metastasis }\end{array}$} & \multirow{2}{*}{ Weight } & \multirow{2}{*}{$\begin{array}{c}\text { Odds ratio } \\
\text { M-H, fixed, } 95 \% \text { CI }\end{array}$} & \multirow{2}{*}{\multicolumn{4}{|c|}{$\begin{array}{c}\text { Odds ratio } \\
\text { M-H, fixed, } 95 \% \text { CI }\end{array}$}} & \\
\hline & Events & Total & Events & Total & & & & & & & \\
\hline \multirow{2}{*}{$\begin{array}{l}\text { Zhang et al., } 2006 \\
\text { Wang et al., } 2012\end{array}$} & 4 & 6 & 34 & 76 & $55.2 \%$ & $2.47[0.43,14.31]$ & & & & ta & \\
\hline & 12 & 13 & 183 & 259 & $44.8 \%$ & $4.98[0.64,39.00]$ & & & & $\rightarrow$ & \\
\hline \multirow{3}{*}{$\begin{array}{l}\text { Total (95\% CI) } \\
\text { Total events } \\
\text { Heterogeneity: } \chi^{2}=\end{array}$} & & 19 & & 335 & $100.0 \%$ & $3.60[0.96,13.53]$ & & & & & \\
\hline & 16 & & 217 & & & & & & & & \\
\hline & $7 ; \mathrm{df}=1$ & $(P=0$ & )$; I^{2}=0$ & & & & 0.01 & 0.1 & 1 & 10 & 100 \\
\hline \multicolumn{5}{|c|}{ Test for overall effect: $Z=1.89(P=0.08)$} & & & & ode $n$ & & No lymph noc & astasis \\
\hline
\end{tabular}

(e)

Figure 6: Continued. 




(f)

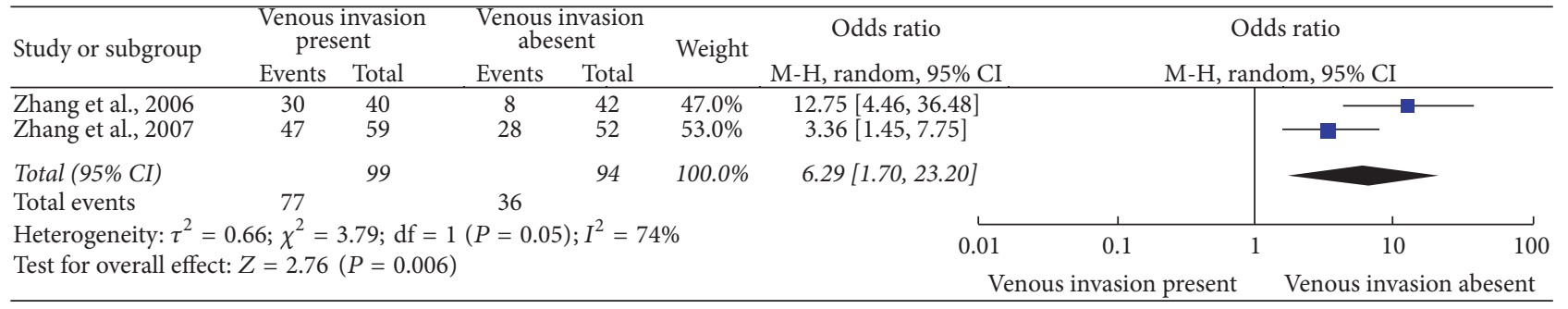

(g)

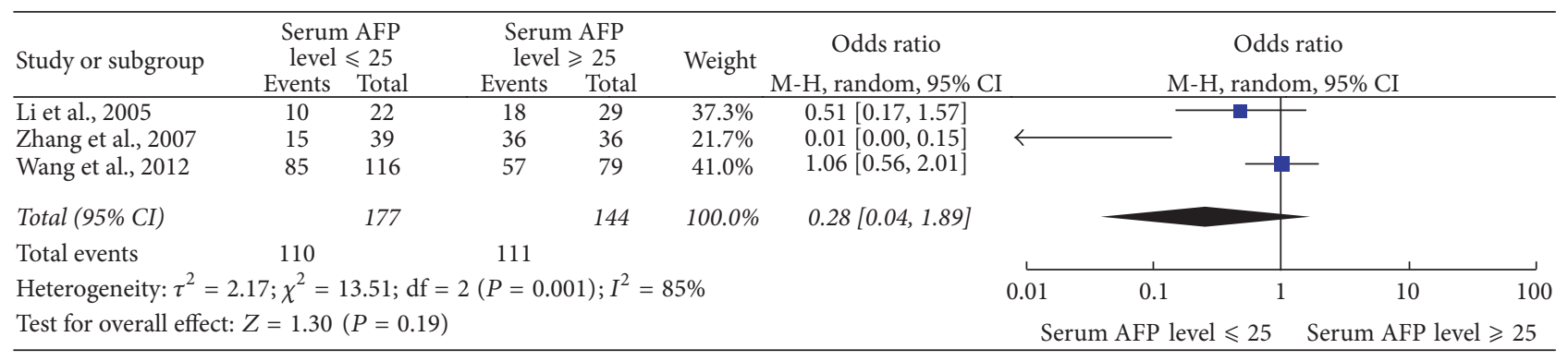

(h)

FIGURE 6: Association of CD147 expression with clinicopathological parameters. Notes. (a) The forest plot for the overall association between CD147 expression and tumor stage in patients with HCC. CD147 expression was not associated with tumor size of HCC cancer (OR = 0.38; 95\% CI: 0.14-1.03; $P=0.06$ ). (b) The forest plot for the overall association between CD147 expression and cirrhosis in patients with HCC. CD147 expression was not associated with cirrhosis of $\mathrm{HCC}$ cancer $(\mathrm{OR}=0.4 ; 95 \% \mathrm{CI}: 0.13-1.45 ; P=0.18)$. (c) The forest plot for the overall association between CD147 expression and differentiation in patients with HCC. CD147 expression was not associated with differentiation of HCC cancer $(\mathrm{OR}=0.54 ; 95 \% \mathrm{CI}: 0.15-1.96 ; P=0.35)$. (d) The forest plot for the overall association between CD147 expression and TMN in patients with HCC. CD147 expression was associated with the TNM stage of HCC cancer (OR $=0.18$; 95\% CI: $0.04-0.85 ; P=0.03)$. (e) The forest plot for the overall association between CD147 expression and lymph node metastasis in patients with HCC. CD147 expression was not associated with lymph node metastasis of HCC cancer (OR $=3.60 ; 95 \% \mathrm{CI}: 0.96-13.53 ; P=0.06)$. (f) The forest plot for the overall association between CD147 expression and HBsAg in patients with HCC. CD147 expression was not associated with HBsAg of HCC cancer $(\mathrm{OR}=1.06$; 95\% CI: $0.64-1.74 ; P=0.82)$. (g) The forest plot for the overall association between CD147 expression and venous invasion in patients with HCC. CD147 expression was associated with venous invasion of HCC cancer $(\mathrm{OR}=6.29 ; 95 \% \mathrm{CI}: 1.70-23.20 ; P=0.006)$. (h) The forest plot for the overall association between CD147 expression and serum AFP level in patients with HCC. CD147 expression was not associated with serum AFP level of HCC cancer (OR = 0.28; 95\% CI: 0.04-1.89; $P=0.19$ ).

multivariate analysis groups, groups with follow-up time less than 5 years, and with liver transplantation. However, further studies are warranted to extend the significance of these results. Our results indicate that patients with low CD147 expression have longer survival time than those with low CD147 expression with huge heterogeneity $\left(I^{2}=92.1 \%\right.$; $P=$ $0.000)$. All three reports $[29,30,34]$ indicated that high CD147 expression group had a shorter median survival time as compared to the low CD147 expression group.
OR for the TNM stage and venous invasion were statistically significant in the correlation study of CD147 expression with the clinical characteristics of patients. Although Zhang et al. [28] and Wang et al. [29] also reported that CD147 expression was closely related to the TNM stage, the results are speculative due to large heterogeneity $\left(I^{2}=91 \% ; P<\right.$ 0.00001). Moreover, $\mathrm{Li}$ [30] also demonstrated that the expression of CD147 was not associated with serum AFP level, tumor size, and differentiation. 
In addition, Tsai et al. [33] demonstrated that the survival rate of the group with EMMPRIN score $\geq 200$ was not significantly different from that of the group with EMMPRIN score $<200(P=0.35)$. Another study by Ji [32] found that there was no significant difference between high and low CD147 expression groups and mean survival time of RFS. In addition, Lee et al. [20] indicated that only the group with sCD147 levels $>24 \mathrm{ng} / \mathrm{mL}$ has a significant difference in 90day survival and 180-day survival compared to sCD147 levels $\leq 24 \mathrm{ng} / \mathrm{mL}$.

It should be noted that there are some limitations to the analyses presented here. Firstly, publication bias can be a concern because more positive results tend to get published, thus potentially exaggerating the association between CD147 expression and poor outcomes. Secondly, in the metaanalysis, HRs and 95\% CI were directly extracted from original data from the three included studies. For other studies, HR had to be extrapolated from the survival curve, implying that the estimated HR may be less reliable than when directly obtained from published statistics. Thirdly, the studies have subjects of different age, follow-up time, and cut-off values. In addition, all patients in these included studies were of Asian origin. Moreover, the quality of some of the included studies was not completely satisfactory. These factors could also have affected the outcome of our evaluation of the prognostic value of CD147.

\section{Conclusion}

Despite the limitations of the present study and heterogeneity across the included studies, our systematic review and metaanalysis suggest that high CD147 expression may be related to the survival, TNM stage, and venous invasion in patients with HCC.

\section{Competing Interests}

The authors declare that they have no competing interests.

\section{Authors' Contributions}

Fei Peng and Hui Li contributed equally to this work.

\section{References}

[1] J. Ferlay, H.-R. Shin, F. Bray, D. Forman, C. Mathers, and D. M. Parkin, "Estimates of worldwide burden of cancer in 2008: GLOBOCAN 2008," International Journal of Cancer, vol. 127, no. 12, pp. 2893-2917, 2010.

[2] R. Siegel, D. Naishadham, and A. Jemal, "Cancer statistics, 2013," CA Cancer Journal for Clinicians, vol. 63, no. 1, pp. 11-30, 2013.

[3] P. A. Farazi and R. A. DePinho, "Hepatocellular carcinoma pathogenesis: from genes to environment," Nature Reviews Cancer, vol. 6, no. 9, pp. 674-687, 2006.

[4] American Cancer Society, Cancer Facts and FIGS 2005, American Cancer Society, 2005, http://www.cancer.org/docroot/ home/index.asp.
[5] Y. Hou, Q. Zou, R. Ge, F. Shen, and Y. Wang, "The critical role

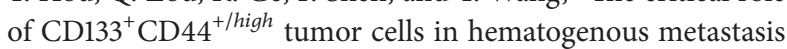
of liver cancers," Cell Research, vol. 22, no. 1, pp. 259-272, 2012.

[6] K. H. Tang, S. Ma, T. K. Lee et al., "CD133 + liver tumorinitiating cells promote tumor angiogenesis, growth, and selfrenewal through neurotensin/interleukin-8/CXCL1 signaling," Hepatology, vol. 55, no. 3, pp. 807-820, 2012.

[7] H. Kim, G. H. Choi, D. C. Na et al., "Human hepatocellular carcinomas with "Stemness"-related marker expression: keratin 19 expression and a poor prognosis," Hepatology, vol. 54, no. 5, pp. 1707-1717, 2011.

[8] O. Waidmann, V. Köberle, D. Bettinger et al., "Diagnostic and prognostic significance of cell death and macrophage activation markers in patients with hepatocellular carcinoma," Journal of Hepatology, vol. 59, no. 4, pp. 769-779, 2013.

[9] T. Kanekura, T. Miyauchi, M. Tashiro, and T. Muramatsu, "Basigin, a new member of the immunoglobulin superfamily: genes in different mammalian species, glycosylation changes in the molecule from adult organs and possible variation in the Nterminal sequences," Cell Structure and Function, vol. 16, no. 1, pp. 23-30, 1991.

[10] J. L. Jiang, Q. Zhou, M. K. Yu, L. S. Ho, Z. N. Chen, and H. C. Chan, "The involvement of HAb18G/CD147 in regulation of store-operated calcium entry and metastasis of human hepatoma cells," Journal of Biological Chemistry, vol. 276, no. 50, pp. 46870-46877, 2001.

[11] C. Biswas, Y. Zhang, R. DeCastro et al., "The human tumor cellderived collagenase stimulatory factor (renamed EMMPRIN) is a member of the immunoglobulin superfamily," Cancer Research, vol. 55, no. 2, pp. 434-439, 1995.

[12] Y. Li, J. Xu, L. Chen et al., "HAb18G (CD147), a cancer-associated biomarker and its role in cancer detection," Histopathology, vol. 54, no. 6, pp. 677-687, 2009.

[13] M. Yang, Y. Yuan, H. Zhang et al., "Prognostic significance of CD147 in patients with glioblastoma," Journal of NeuroOncology, vol. 115, no. 1, pp. 19-26, 2013.

[14] J. L. Jiang, H. C. Chan, Q. Zhou et al., "HAb18G/CD147mediated calcium mobilization and hepatoma metastasis require both C-terminal and N-terminal domains," Cellular and Molecular Life Sciences, vol. 61, no. 16, pp. 2083-2091, 2004.

[15] T. Kanekura, X. Chen, and T. Kanzaki, "Basigin (CD147) is expressed on melanoma cells and induces tumor cell invasion by stimulating production of matrix metalloproteinases by fibroblasts," International Journal of Cancer, vol. 99, no. 4, pp. 520-528, 2002.

[16] J. Sun and M. E. Hemler, "Regulation of MMP-1 and MMP2 production through CD147/extracellular matrix metalloproteinase inducer interactions," Cancer Research, vol. 61, no. 5, pp. 2276-2281, 2001.

[17] J. Xu, H.-Y. Xu, Q. Zhang et al., "HAb18G/CD147 functions in invasion and metastasis of hepatocellular carcinoma," Molecular Cancer Research, vol. 5, no. 6, pp. 605-614, 2007.

[18] S. Zhu, Y. Li, Y. Zhang et al., "Expression and clinical implications of HAb18G/CD147 in hepatocellular carcinoma," Hepatology Research, vol. 45, no. 1, pp. 97-106, 2015.

[19] L. Xiong, C. Edwards, and L. Zhou, "The biological function and clinical utilization of CD147 in human diseases: a review of the current scientific literature," International Journal of Molecular Sciences, vol. 15, no. 10, pp. 17411-17441, 2014.

[20] A. Lee, A. Rode, A. Nicoll et al., "Circulating CD147 predicts mortality in advanced hepatocellular carcinoma," Journal of 
Gastroenterology and Hepatology (Australia), vol. 31, no. 2, pp. 459-466, 2016.

[21] Q. Zhang, J. Zhou, X.-M. Ku et al., "Expression of CD147 as a significantly unfavorable prognostic factor in hepatocellular carcinoma," European Journal of Cancer Prevention, vol. 16, no. 3, pp. 196-202, 2007.

[22] A. Stang, "Critical evaluation of the Newcastle-Ottawa scale for the assessment of the quality of nonrandomized studies in metaanalyses," European Journal of Epidemiology, vol. 25, no. 9, pp. 603-605, 2010.

[23] M. K. B. Parmar, V. Torri, and L. Stewart, "Extracting summary statistics to perform meta-analyses of the published literature for survival endpoints," Statistics in Medicine, vol. 17, no. 24, pp. 2815-2834, 1998.

[24] J. F. Tierney, L. A. Stewart, D. Ghersi, S. Burdett, and M. R. Sydes, "Practical methods for incorporating summary time-toevent data into meta-analysis," Trials, vol. 8, article 16, 2007.

[25] P. R. Williamson, C. T. Smith, J. L. Hutton, and A. G. Marson, "Aggregate data meta-analysis with time-to-event outcomes," Statistics in Medicine, vol. 21, no. 22, pp. 3337-3351, 2002.

[26] S. Duval and R. Tweedie, "Trim and fill: a simple funnel-plotbased method of testing and adjusting for publication bias in meta-analysis," Biometrics, vol. 56, no. 2, pp. 455-463, 2000.

[27] H.-G. Li, D.-R. Xie, X.-M. Shen, H.-H. Li, H. Zeng, and Y.-J. Zeng, "Clinicopathological significance of expression of paxillin, syndecan-1 and EMMPRIN in hepatocellular carcinoma," World Journal of Gastroenterology, vol. 11, no. 10, pp. 1445-1451, 2005.

[28] Q. Zhang, X. Chen, J. Zhou et al., “CD147, MMP-2, MMP-9 and MVD-CD34 are significant predictors of recurrence after liver transplantation in hepatocellular carcinoma patients," Cancer Biology \& Therapy, vol. 5, no. 7, pp. 808-814, 2006.

[29] W. Wang, M. Zhao, and Y. Li, "Expressions and clinical significance of CD147 and CK19 in hepatocellular carcinoma," Chinese-German Journal of Clinical Oncology, vol. 11, no. 9, pp. 517-521, 2012.

[30] Z. Y. Li, Expression and Clinical Significance of MMP-9 and CD147 in Human Hepatocellula Carcinoma, Zhejiang University Medical College, 2010.

[31] M. Luo, Expression of CD147 in Hepatocellular Carcinoma and Its Significance on Posttransplant Prognostic Patients, Niaoling Medical University, 2011.

[32] S. P. Ji, Expression and Clinical Significance of TIMP-2, MMP2 and CD147 in Hepatocellular Carcinoma, Tianjin Medical University, 2011.

[33] W.-C. Tsai, Y.-C. Chao, W.-H. Lee, A. Chen, L.-F. Sheu, and J.-S. Jin, "Increasing EMMPRIN and matriptase expression in hepatocellular carcinoma: tissue microarray analysis of immunohistochemical scores with clinicopathological parameters," Histopathology, vol. 49, no. 4, pp. 388-395, 2006.

[34] L. F. Zhang, The Impact of CD147 and Klotho on the Prognosis of Patients with Hepatocellular Carcinoma, Central South University, 2014.

[35] J. Ferlay, I. Soerjomataram, R. Dikshit et al., "Cancer incidence and mortality worldwide: sources, methods and major patterns in GLOBOCAN 2012," International Journal of Cancer, vol. 136, no. 5, pp. E359-E386, 2015.

[36] A. Caudron, M. Battistella, J.-P. Feugeas et al., "EMMPRIN/CD147 is an independent prognostic biomarker in cutaneous melanoma," Experimental Dermatology, vol. 25, no. 8, pp. 618-622, 2016.
[37] T. M. Bauman, J. A. Ewald, W. Huang, and W. A. Ricke, "CD147 expression predicts biochemical recurrence after prostatectomy independent of histologic and pathologic features," BMC Cancer, vol. 15, no. 1, article 549, 2015. 


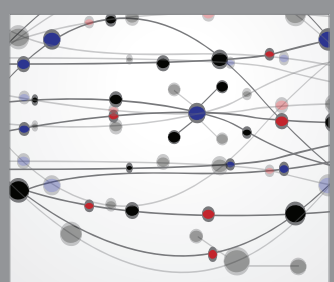

The Scientific World Journal
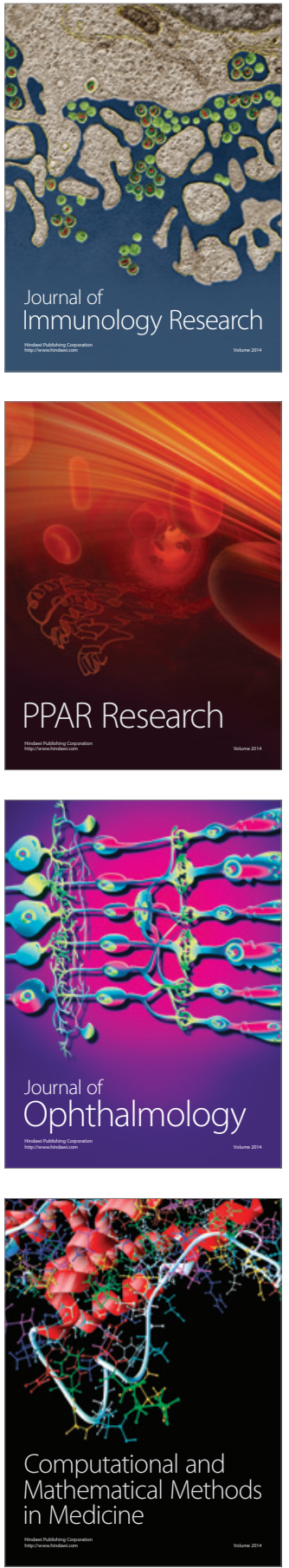

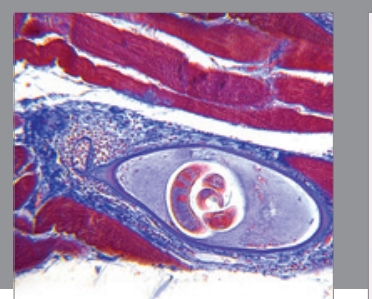

Gastroenterology Research and Practice


Disease Markers

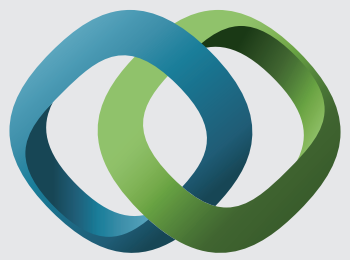

\section{Hindawi}

Submit your manuscripts at

https://www.hindawi.com
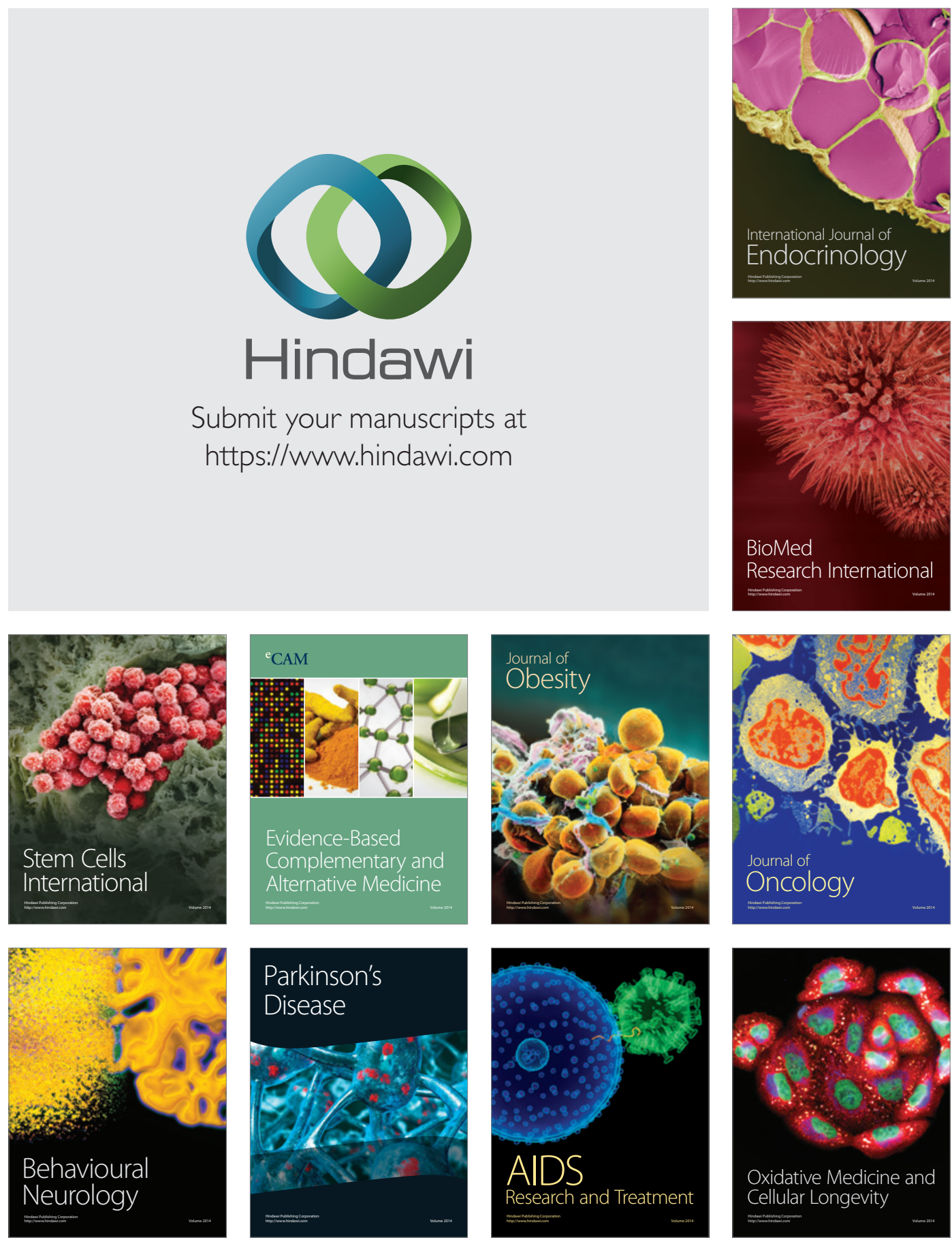